\title{
Antigen-Presenting Cells: Potential of Proven und New Players in Immune Therapies
}

\author{
Britta Eiz-Vesper ${ }^{\mathrm{a}}$ Helga Maria Schmetzer ${ }^{\mathrm{b}}$ \\ anstitute of Transfusion Medicine and Transplant Engineering, Hannover Medical School, Hannover, Germany; \\ ${ }^{b}$ Med III, Department for Hematopoietic Transplantations, University Hospital of Munich - Grosshadern, Munich, \\ Germany
}

The immune system is permanently confronted with mutated and self-, microbe-, and tumor-derived neoantigens - as well as other, "unknown" antigens - and has to differentiate between self or nonself. These antigenic molecules (protein or lipid based) must be phagocytosed, processed, and/or presented in the respective major histocompatibility complex (MHC) molecules on the cell surface in recognizable form to train immune cells such as effector $\mathrm{T}$ cells, leading to their specific activation. These "trainers" are so-called antigen-presenting cells (APCs), which can be divided into professional (e.g., dendritic cells [DCs], B cells, and macrophages) and nonprofessional APCs (e.g., fibroblasts and hepatocytes). While all nucleated human cells can present peptide fragments of endogenous proteins using the MHC class I pathway and display them on the surface to CD8+ cytotoxic T lymphocytes [1-3], only professional APCs such as DCs, macrophages, and $\mathrm{B}$ cells are characterized by the ability to present exogenous antigens using MHC class II molecules and present them on MHC class II molecules to CD4+ T-helper cells ( $\mathrm{T}_{\mathrm{H}}$ cells), along with the required costimulatory molecules, such as CD86 and CD83 molecules [2]. Therefore, the main difference between professional and nonprofessional APCs is the absence of MHC class II and costimulatory molecules on nonprofessional APCs. Recently, it was described that the three main granulocyte subsets (neutrophils, eosinophils, and basophils) also seem to be able to present exogenous antigens to naive $\mathrm{T}_{\mathrm{H}}$ cells via $\mathrm{MHC}$ class II molecules, which has led to the suggestion that they should be referred to as APCs $[4,5]$.
Only professional APCs provide all three signals (antigen presentation via $\mathrm{MHC}$ molecules, expression of costimulatory molecules, and cytokine/chemokine secretion) needed to train and activate $\mathrm{T}$ cells to recognize, destroy, or tolerate cells that carry these antigens, and thereby to control viral infections or cancer cell growth $[6,7]$. Macrophages and DCs internalize pathogens and cellular debris by phagocytosis, whereas B cells use the Bcell receptor for antigen uptake. Antigens are presented to $\mathrm{T}$ cells along with the required costimulatory molecules to get activated, get "licensed" to mediate their (helper or cytotoxic) function, and produce memory cells.

DCs are most effective at presenting tumor and viral antigens of intracellular origin because they have the ability to "cross-present" antigens [8]. A variety of DC subtypes in various organs with different phenotypical and functional characteristics mediating wound healing, proinflammation, or anti-infectious or antitumor attack were described, and they can be used for immune profiling to monitor the grade of activation or suppression of the immune system $[9,10]$. Plasmacytoid or special tolerogenic DCs regulate responses of the innate and adaptive immune cells and contribute to avoiding autoimmune reactions [10-12].

DC-based treatments have been applied for almost three decades and so far have been tested most often in patients with malignant melanoma, prostate cancer, malignant glioma, or renal cell cancer [13, 14]. DCs were also applied in combination with cytokine-induced killer cells to treat gastrointestinal tumors, lung cancer, and breast 
cancer, as well as hematological malignancies [15]. Their clinical effectiveness was markedly increased after methods for generation, cultivation, and manipulation to exploit the immune-activating potential of DCs had been improved $[14,16]$. The protocols were substantially improved with respect to optimization and standardization to increase cell yield, reduction of culture time, the differentiation process, and antigen loading. Recently, it was described that reprogramming of monocytes with lentiviral vectors expressing granulocyte-macrophage colony-stimulating factor (GM-CSF), interferon (IFN)- $\alpha$, interleukin (IL)-4, and antigens will lead to maturation of DCs and the induction of autocrine and paracrine immune effects against virus- or leukemia-associated antigens [17, 18].

A specialty of myeloid leukemic blasts is their capability to differentiate into "leukemia-derived DCs" [19-22]. Those DCs can either be generated ex vivo or used for adoptive transfer to patients with leukemia. Alternatively, blasts in the body can be converted into leukemia-derived DCs after treatment with approved drugs. This strategy is successful independent of patients' leukemic subtype, mutation, MHC expression, or transplantation status.

Macrophages, derived from the same progenitor cells as DCs, express MHC class II and costimulatory molecules after activation by IFN- $\gamma$. They also circulate in the blood and enter sites of infections or tissue damage and have been shown to be involved in cross-presentation of antigens [23]. Macrophages can be generated in vitro from monocytes or $\mathrm{CD} 34$ progenitor cells in the presence of cytokines such as M-CSF and GM-CSF, but a great heterogeneity in origin and tissue-specific functions was found, making the standardization of protocols more difficult. Protocols for generating APCs and macrophages as off-the-shelf products from induced pluripotent stem cells are under investigation, and they might open up a new field for generating and designing macrophages on a large scale to be used for clinical studies [24, 25].

To overcome limitations to the generation of conventional APCs - especially for cancer patients, where the functionality of both APCs and effector cells is impaired - alternative strategies are under investigation. Engineered MHC class I-deficient K562 cells or paramagnetic nanoparticle-based artificial (a) APCs were designed to optimize and control T-cell signals required for activation, expansion, and costimulation via human leukocyte antigen-restricted peptide complex and costimulatory signals $[26,27]$. These tools make the generation of APCs cost-effective, highly reproducible, and scalable, and generated $\mathrm{T}$-cell products will be capable of generating potent and durable responses in treated patients [2830]. Interestingly, genetically modified K562-based aAPCs were recently used as an inexhaustible source for CD19-directed chimeric antigen receptor (CAR) T-cell expansion, thereby opening up new areas of APC applica-

tion [31]. This approach was found to be less prone to variability in CAR T-cell expansion than a standard beadbased approach, and it resulted in CAR T cells with potent antitumor responses in preclinical models of acute lymphoblastic leukemia and B-cell lymphoma.

The knowledge of APCs' biology, their function and regulation, and their role in a pathological context (e.g., during the course of infection, allergy, autoimmunity, transplant rejection, or tumor immunological processes) is indispensable when utilizing APCs for clinical applications. In summary, highly professional APCs for cellular therapies can be generated or addressed by

- optimization of culture conditions for APCs and production under GMP (Good Manufacturing Practice) conditions [16, 22];

- generation of new, highly specialized APCs after loading with tumor antigens or generation of leukemiaderived APCs from myeloid blasts ex vivo [16];

- in vivo production of leukemia-derived DCs from blasts in the body [22];

- genetic engineering of precursors for APC generation $[18,25]$;

- usage of off-the-shelf APCs (DCs or macrophages) generated from induced pluripotent stem cells [25]; and

- establishment of aAPCs such as nanoparticles under highly reproducible conditions [30].

Our understanding of the mechanisms involved in antigen processing and presentation will be leading to effective and durable cellular and humoral immune responses and will contribute to the design of new vaccination strategies against microbial or tumor targets, to refinement of immune monitoring, to blockage of APC-mediated "overactivation" of the immune system, and to the development of attractive and useful accessories for improvement of new cell-based therapies.

References

Transfus Med Hemother 2020;47:429-431 DOI: $10.1159 / 000512729$
1 Hewitt EW. The MHC class I antigen presentation pathway: strategies for viral immune evasion. Immunology. 2003 Oct;110(2):1639.

2 Blum JS, Wearsch PA, Cresswell P. Pathways of antigen processing. Annu Rev Immunol. 2013;31:443-73.

3 Nakayama M. Antigen Presentation by MHC-Dressed Cells. Front Immunol. 2015 Jan;5:672.

4 Kambayashi T, Laufer TM. Atypical MHC class II-expressing antigen-presenting cells: can anything replace a dendritic cell? Nat Rev Immunol. 2014 Nov; 14(11):719-30.

5 Lin A, Loré K. Granulocytes: New Members of the Antigen-Presenting Cell Family. Front Immunol. 2017 Dec;8:1781.

6 Curtsinger JM, Mescher MF. Inflammatory cytokines as a third signal for $\mathrm{T}$ cell activation. Curr Opin Immunol. 2010 Jun;22(3):333-40. 
7 den Haan JM, Arens R, van Zelm MC. The activation of the adaptive immune system: cross-talk between antigen-presenting cells, T cells and B cells. Immunol Lett. 2014 Dec; 162(2 Pt B):103-12.

8 Joffre OP, Segura E, Savina A, Amigorena S. Cross-presentation by dendritic cells. Nat Rev Immunol. 2012 Jul;12(8):557-69.

9 Kushwah R, Hu J. Complexity of dendritic cell subsets and their function in the host immune system. Immunology. 2011 Aug;133(4):40919.

10 Kelly PN. More DC subtypes revealed. Science. 2019;366(6470):1210.

11 Takenaka MC, Quintana FJ. Tolerogenic dendritic cells. Semin Immunopathol. 2017 Feb; 39(2):113-20.

12 Zhuang Q, Cai H, Cao Q, Li Z, Liu S, Ming Y. Tolerogenic Dendritic Cells: The Pearl of Immunotherapy in Organ Transplantation. Front Immunol. 2020 Oct; 11:552988.

13 Anguille S, Smits EL, Lion E, van Tendeloo VF, Berneman ZN. Clinical use of dendritic cells for cancer therapy. Lancet Oncol. 2014 Jun;15(7):e257-67.

14 Patente TA, Pinho MP, Oliveira AA, Evangelista GC, Bergami-Santos PC, Barbuto JA. Human Dendritic Cells: Their Heterogeneity and Clinical Application Potential in Cancer Immunotherapy. Front Immunol. 2019 Jan;9: 3176.

15 Garofano F, Gonzalez-Carmona MA, Skowasch D, Schmidt-Wolf R, Abramian A Hauser S, et al. Clinical Trials with Combination of Cytokine-Induced Killer Cells and Dendritic Cells for Cancer Therapy. Int J Mol Sci. 2019 Sep;20(17):E4307.

16 Cunningham S, Hackstein H. Recent Advances in Good Manufacturing Practice-Grade Generation of Dendritic Cells. Transfus Med Hemother. 2020 [Online ahead of print].
17 Daenthanasanmak A, Salguero G, Borchers S, Figueiredo C, Jacobs R, Sundarasetty BS, et al. Integrase-defective lentiviral vectors encoding cytokines induce differentiation of human dendritic cells and stimulate multivalent immune responses in vitro and in vivo. Vaccine. 2012 Jul;30(34):5118-31.

18 Bialek-Waldmann JK, Heuser M, Ganser A, Stripecke R. Monocytes reprogrammed with lentiviral vectors co-expressing GM-CSF, IFN- $\alpha 2$ and antigens for personalized immune therapy of acute leukemia pre- or poststem cell transplantation. Cancer Immunol Immunother. 2019 Nov;68(11):1891-9.

19 Kremser A, Dressig J, Grabrucker C, Liepert A, Kroell T, Scholl N, et al. Dendritic cells (DCs) can be successfully generated from leukemic blasts in individual patients with AML or MDS: an evaluation of different methods. J Immunother. 2010 Feb-Mar;33(2):185-99.

20 Van Acker HH, Versteven M, Lichtenegger FS, Roex G, Campillo-Davo D, Lion E, et al. Dendritic Cell-Based Immunotherapy of Acute Myeloid Leukemia. J Clin Med. 2019 Apr;8(5):E579.

21 Ansprenger C, Amberger DC, Schmetzer HM. Potential of immunotherapies in the mediation of antileukemic responses for patients with acute myeloid leukemia (AML) and myelodysplastic syndrome (MDS) - with a focus on dendritic cells of leukemic origin (DCleu). Clin Immunol. 2020 Aug;217:108467.

22 Amberger DC, Schmetzer HM. Dendritic Cells of Leukemic Origin - Specialized Antigen Presenting Cells as Potential Treatment Tools for Patients with Myeloid Leukemia. Transfus Med Hemother. 2020 [Online ahead of print].

23 Oishi Y, Manabe I. Macrophages in inflammation, repair and regeneration. Int Immunol. 2018 Oct;30(11):511-28.
24 Lee CZ, Kozaki T, Ginhoux F. Studying tissue macrophages in vitro: are iPSC-derived cells the answer? Nat Rev Immunol. 2018 Nov; 18(11):716-25.

25 Ackermann M, Dragon AC, Lachmann N. The Immune-Modulatory Properties of iPSC-Derived Antigen-Presenting Cells. Transfus Med Hemother. 2020 [Online ahead of print].

26 Thomas AK, Maus MV, Shalaby WS, June $\mathrm{CH}$, Riley JL. A cell-based artificial antigenpresenting cell coated with anti-CD3 and CD28 antibodies enables rapid expansion and long-term growth of CD4 T lymphocytes. Clin Immunol. 2002 Dec;105(3):259-72.

27 Perica K, De León Medero A, Durai M, Chiu YL, Bieler JG, Sibener L, et al. Nanoscale artificial antigen presenting cells for T cell immunotherapy. Nanomedicine. 2014 Jan;10(1): 119-29.

28 Neal LR, Bailey SR, Wyatt MM, Bowers JS, Majchrzak K, Nelson MH, et al. The Basics of Artificial Antigen Presenting Cells in T CellBased Cancer Immunotherapies. J Immunol Res Ther. 2017;2(1):68-79.

29 Ichikawa J, Yoshida T, Isser A, Laino AS, Vassallo M, Woods D, et al. Rapid Expansion of Highly Functional Antigen-Specific T Cells from Patients with Melanoma by Nanoscale Artificial Antigen-Presenting Cells. Clin Cancer Res. 2020 Jul;26(13):3384-96.

30 Suarez L, Wang R, Carmer S, Bednarik D, Myint $\mathrm{H}$, Jones K, et al. AIM Platform: A Novel Nano aAPC-Based Clinical System Designed to Consistently Produce Multi-Antigen Specific T Cell Products with Potent and Durable Anti-tumor Properties. Transfus Med Hemother. 2020 [Online ahead of print].

31 Schmidts A, Marsh LC, Srivastava AA, Bouffard AA, Boroughs AC, Scarfò I, et al. Cellbased artificial APC resistant to lentiviral transduction for efficient generation of CAR$\mathrm{T}$ cells from various cell sources. J Immunother Cancer. 2020 Sep;8(2):e000990. 